\title{
Investigation of Timbral Qualities of Guitar Using Wavelet Analysis
}

\author{
Şafak Ekmen ${ }^{1,2^{*}}$, Can Karadoğan ${ }^{2,3}$, Şahin Serhat Şeker ${ }^{1}$ \\ ${ }^{1}$ Department of Electrical Engineering, Istanbul Technical University, Istanbul 34469, Turkey \\ ${ }^{2}$ Dr. Erol UCER Center for Advanced Studies in Music (MIAM), Istanbul Technical University, Istanbul 34367, Turkey \\ ${ }^{3}$ Turkish Music State Conservatory, Department of Music Technology, Istanbul Technical University, Istanbul 34357, Turkey
}

Corresponding Author Email: ekmen@itu.edu.tr

https://doi.org/10.18280/ts.380218

Received: 24 September 2020

Accepted: 10 February 2021

\section{Keywords:}

wavelet analysis, digital signal processing, continuous wavelet transform, wavelet packet transform, guitar analysis, timbre, piezo-film sensors

\begin{abstract}
Aim of this study is presenting a practical and accurate approach for objective evaluation of guitars that is suitable for performing by the different parties of the field. For this purpose, timbral qualities of classical guitars are investigated using the power of wavelet analysis. A complete system from data capturing to their analysis is proposed. A mass produced guitar that is described as a learning guitar by its production company and a luthier-made guitar are analyzed. Procedure is done with the piezo-film sensors attached to the guitar and the player holding it in a conventional playing position while plucking the strings with a pluck. Continuous Wavelet and Wavelet Packet Transforms are employed for analysis using MATLAB Wavelet Toolbox. High resolution results show detailed presentation of harmonic and inharmonic partials as well as time envelopes of them. This allows for objective analysis on the timbre of a guitar as well as making comparison between the timbral properties of two guitars.
\end{abstract}

\section{INTRODUCTION}

Instrument making is a traditionally empirical process which, relies on the ears of instrument makers and players. Most of the design approaches that are being used today was invented as back as 18 th century by Anthony Torres Juredo. The most popular version we use today has six strings. These are tuned to the pitches E2, A2, D3, G3, B3 and E4 with the frequencies of $82 \mathrm{~Hz}, 110 \mathrm{~Hz}, 147 \mathrm{~Hz}, 196 \mathrm{~Hz}, 247 \mathrm{~Hz}$ and $330 \mathrm{~Hz}$ respectively. Strings are tied to tune heads at the one end and to the bridge on the other in a parallel position to each other. Classical guitars, such as the ones used in this study, are acoustic instruments with nylon strings. The main energy of sound generated by guitar comes from its body. If one traces the signal generated by the vibration of strings; it can be seen that vibrations of top plate, back plate and air cavity as well as their coupling contributes with different weights and in different frequencies to the final sound radiating from the guitar. Design parameters that have the most effect on the sound characteristics of a guitar is: bracing, size and the choice of wood. How they are being assembled coming second [1].

Most audio equipment has their full specifications including frequency response available. Therefore, a buyer can have a rough idea about the sound of the equipment. This is because there is considerable research on the categorization of their design parameters to their characteristics. On the other hand, even though one can also obtain the full specifications of a particular instrument, they still have a hard time to imagine its sound and to compare with its counterparts. Thus, most end users, especially the ones that are inexperienced, still have to lean on the reviews of others. It is not uncommon to come across questions like "Is this guitar have these characteristics?" or "Is this guitar have that characteristics which is important for that particular genre?" Also, it is no secret that not everyone has the financial means of obtaining a carefully handmade instrument from a renowned luthier or obtaining a build that is well established. So, information on the characteristics become more and more important.

One of the most established technique for studying characteristics of guitars is using Chladni patterns [2]. Invented by physicist Ernst Chladni, it is for determining the vibration modes of the plates made from different materials. After spreading a low density material in dust form on the plate in focus, it is excited by an external force and the patterns the dust creates is observed. It can be understood that this method is rather cumbersome. Luckily, promising advancements have been made on the topic over the past fifty years. In Rossing's study, holographic interferometry is used [3]. In Curtu's study resonance frequencies and modal vibrations of the guitar body (without the neck attached to it) are investigated using Chladni patterns with the addition of accelerometers, captured data is analyzed using Fast Fourier Transform (FFT) [4]. In Patil's study, Digital Image Correlation was used [5]. A freely supported guitar is excited with a sound source and resulting vibrations captured with high speed cameras. As a result, FFT of these are presented. Woodhouse [6] studied the transients of modal frequencies that are belong to the string, guitar body and the coupling of two. Plucking was used as excitation, data is captured again by accelerometers and analysis is performed using Short Time Fourier Transform (STFT). Researchers justify this choice by stating that time-frequency techniques are more suitable because, especially transients of separate frequency components contain important information and these components are usually close to each other. Approaches in above studies have common disadvantages: (i) their testing setups are hard to install (ii) FFT doesn't provide any timefrequency localization (iii) STFT has low resolution and doesn't have the adequate flexibility for detailed analysis of 
sound signals. As a result, none of these techniques found a popular place among the independent instrument makers. Therefore, completely constructing the instrument and evaluating by ear is still a wide choice.

This study presents a practical and accurate approach for investigating timbral qualities of classical guitars. Proposed system includes: a relatively effortless test setup, data capturing with piezoelectric film sensors and analysis using wavelet transform. The approach can be used by the different parties of the field: from instrument makers and designers to the quality control engineers and end users for the objective evaluation. Moreover, it is believed that with the application specific improvements, the system can be used in wide range of applications such as: detailed cataloging of characteristics of different guitars, music information retrieval and probably even for Genuity tests.

\section{TIMBRE}

There is no exhaustive definition for timbre. One of the most used and accepted one is by American National Standards Institute [7], which, points to the difference of two sounds having the equal loudness and pitch as they perceived by a listener. It attributes this difference to the spectrum content of the signal and their time-varying properties. Richardson [8] shows the effect of transients on an instrument's timbre by investigating the recordings of wind instruments. It also presents that the content of transient and steady state portion is different. Berger [9] presents the same phenomenon with addition of the decay part by modifying aforementioned portions and conducting listening experiments. Craig and Jeffress [10] uses two pure tones and combine them with different phase relations in order to demonstrate that the effect of time difference between the frequency components is important. In Grey' work, researches for a compact criteria space for the recognition of timbre and presents the following properties: distribution of energy among the spectrum including inharmonic partials; amount of energy in the attack time, phase relationship especially between the transient portions of high frequency components and again timevariation of frequency components [11]. As it can be understood, spectral content being the foundation of timbre, envelope of this content is also important.

Much like any object, instruments have natural frequencies. If an instrument is excited with the harmonics of these, it results with sympathetic vibrations. Natural frequencies are determined by the build and material choice and they will be present in the radiating sound of the instrument independently from the content it is excited [12]. Thus, non-integer harmonics of a played pitch created by the acoustical qualities of the instrument have an important effect on its timbre.

\section{WAVELET THEORY AND ITS MUSICAL APPLICATIONS}

\subsection{Wavelet theory}

Mathematical transforms can be considered as looking same information with a different viewpoint [13]. To define the transformations, first the inner product should be defined:

$$
\langle x(t), g(t)\rangle=\int x(t) g(t) d t
$$

where, $\mathrm{g}(\mathrm{t})$ is the basis function and can be considered as the analyzing tool. In Fourier Transform, input signal is decomposed to sine and cosine basis functions and transformation is performed over the complete duration of signal at once [14]. Thus, time information of the frequency components is not presented. To obtain this, "Windowed Fourier Transform" or "Short Time Fourier Transform (STFT)" is developed. In STFT, basis function is constrained with a fixed size window. The size of the window is defined by time resolution $\Delta t$ and frequency resolution $\Delta f$. This size which is the resolution of analysis is restricted in practice with the Heisenberg's Uncertainty principle:

$$
\Delta t \Delta f \geq \frac{1}{4 \pi}
$$

It is discovered by Gabor [15] that when Gaussian window functions are used, Eq. (2) becomes an equality. However, using fixed size windows is a disadvantage because, a real world sound signal consists of both low and high frequency components and fixed-size windows are lack the accuracy in either one of them. The first logical solution to that is using varying sized windows. So, if we denote $\psi(t)$ to a window (basis) function with a variable size, call it wavelet and define this basis function as:

$$
\psi_{a, b}(t)=\frac{1}{\sqrt{a}}\left(\frac{t-b}{a}\right)
$$

where, " $a$ " is the dilation and " $b "$ is the translation parameter; the transform becomes the Continuous Wavelet Transform (CWT). CWT is computed for infinite number of translations which is impractical. Also, shifts of the wavelet with infinitely small intervals, results in overlaps which, creates a high redundancy. To overcome this, dilation and translation parameters can be discretized in order to compute CWT. But, even with this way redundancy is still present and the perfect inversion is impossible because of the phenomenon known as “aliasing". True Discrete Wavelet Transform (DWT) is mainly developed by Meyer [16] and Mallat [17]. Meyer found the orthogonality of wavelets. Orthogonality can simply be defined as all of the basis functions of a vector space satisfying the following equality: let $V_{1}$ and $V_{2}$ are functions (or vectors) of a vector space $V$ then,

$$
\left\langle V_{1}, V_{2}\right\rangle=0
$$

This allows performing a non-redundant wavelet transform. Mallat added that a signal can be decomposed to orthonormal subspaces if orthonormal wavelets are used. This way, using a sequence of scaled versions of mother wavelets, signals can be analyzed to a desired level that can be thought as resolution. This technique makes wavelets similar to high and low pass filters and removes the necessity of tuning the parameters which makes adaptive analysis possible. Mallat named this technique "Multi-Resolution Analysis (MRA)". Later, Daubechies developed compactly supported wavelets with the highest number of vanishing points [18-20]. In simple terms, these are zero outside of their defined time interval and have the highest time-frequency localization. Daubechies Wavelets can be used both in DWT and in MRA.

In MRA, sequential filters are applied only to output of the low pass filter at the preceding level. This is a disadvantage when it is performed on signals that have information spread 
out through their whole frequency range; such as sound signals. Meyer et al. introduced "Wavelet Packet Transform (WPT)" which, filters are applied also to the output of the high-pass filter at the preceding level so it creates a tree structured analyzing scheme [21-23]. As a result, WPT got a place between CWT and MRA in following sense: WPT can be perfectly constructed and computationally more efficient then CWT while having higher resolution than MRA.

\subsection{Musical applications of wavelet theory}

Literature on the application of wavelet analysis to musical signals, supports the necessity of it for the analysis of timbral qualities. Alm and Walker [24] present that musical instrument sounds are non-stationary which requires a form of timefrequency representation. They consist of even and odd partials which are especially crowded in low-frequency region which makes a high-resolution analysis necessary. Since these low partials are mostly resulted from the body of the instrument, they are important for the analysis of timbre. Moreover, spectrum of musical signals is distributed in base 2 logarithmic scale which is called "octave"; combining this with the fact that they have important information on transients, they point that wavelet analysis is more suitable and preferable to a fixed-size transformation such as STFT. This can be justified by looking at the studies on timbre in Section 2 and the information on STFT above. While making similar remarks with the previous, Pielemeier et al. [25] also proposes Empirical-Mode Decompositions which are different group of techniques for time-frequency presentation. Salimpour and Abolhassani [26] perform a wavelet transform algorithm based on the model of perception of human's ear. Dutilleux et al. are also demonstrated musical applications of wavelet analysis [27-29].

\section{EXPERIMENT AND ANALYSIS}

\subsection{Experiment}

Experiments was performed in the main studio of Istanbul Technical University/Center of Advance Studies in Music. Two guitars were used: a mass produced guitar that is described as a learning guitar by its production company and a luthier-made guitar. Procedure is done while a guitar player holding the guitar in a conventional playing position and picking the strings with a pluck. A photograph showing the player while holding the guitar is presented in Figure 1.

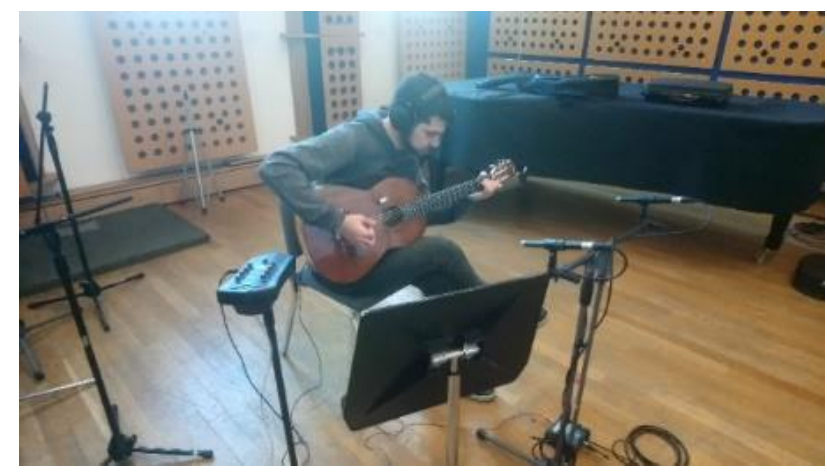

Figure 1. Holding position
A minor diatonic scale with addition of A minor chord at the end, both in open position and 5th position is played. Choice of the played scale was rather arbitrary whereas the choice of playing in two different positions is made to observe the effect of excitation epicenter on the fingerboard. Each material is played at least 5 times to obtain the most consistent data.

Piezo-film sensors, specifically two SDT-028K are used because, as stated in their datasheet [30], they have the frequency range between $10 \mathrm{~Hz}$ to $100 \mathrm{kHz}$ as well as having near-flat frequency response. Most importantly, mass of the piezo-film sensor is much lower than their main competitor accelerometers [31]. This makes it possible to neglect their effect on natural resonance of the guitar body. Piezoelectric film sensors are capacitive films; vibrations of the surface they are attached on creates pressure on the film and in turn the film creates electric potential as a result of electrostatic field. The choice on the number of sensors used in the experiment is bounded by financial limits. Sensors are modified with cables having the gauge around $20 \mathrm{AWG}$ to be able to place electrical end in a comfortable distance. For the connection to the amplifier system, $6.35 \mathrm{~mm}$ TRS plug was soldered to the open ends of the cables. One sensor is placed near the air cavity in a parallel fashion and the other placed under the bridge in parallel. Figure 2 shows the placement of sensors. The placement is based on the common points of vibrations that are observed in the aforementioned studies. These areas are at the top and bottom sides of the bridge and a lung shaped area around the air cavity. Although intensity of vibrations on the top of air cavity is greater, placing the sensor on the bottom part could be more convenient because of the playing position. To attach the sensors, non-foam double sided tapes were used as recommended in their datasheet. When choosing the tape, not harming the coating on the wood but safely carrying the sensors was the consideration. It has been seen that the tapes had not damaged the coating in both guitars. Sensors are connected to isolation transformers. In the datasheet, it is stated the input impedance of the transformer should be between $1 \mathrm{MOhm}$ and $10 \mathrm{MOhm}$ for the appropriate power transfer and if the $1 \mathrm{MOhm}$ is used there will be $6 \mathrm{~dB}$ attenuation in the range of $50 \mathrm{~Hz}$ to $100 \mathrm{~Hz}$. Because the transformers ready at hand has the maximum impedance of 1 $\mathrm{MOhm}$ and the attenuation in the stated frequency range is considered to be negligible, this setting was used. Output of the transformers are connected to professional grade audio preamplifiers and signals are recorded through an audio interface with 24 bit bit-depth and $44.1 \mathrm{kHz}$ sampling rate. Signal flow of the experiment can be seen in Figure 3 .

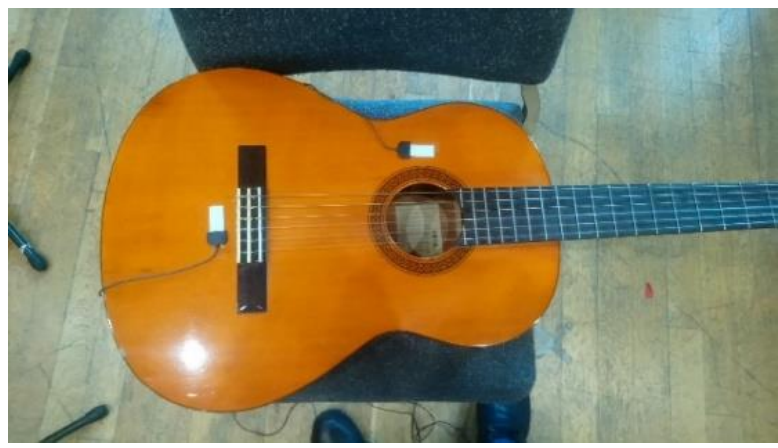

Figure 2. Sensor placement 


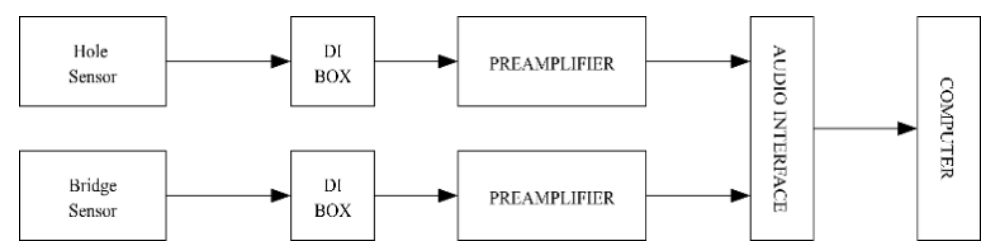

Figure 3. Signal flow

\subsection{Analysis}

Analyzing all of the captured data would far exceed the time limitations of this study. Therefore, samples of A2, A3, A4 pitches at the both fingerboard positions and from the both sensors are selected. Choice was made to cover the most of the frequency range of guitar while keeping the variable at minimum. Because priori knowledge about the researched properties is existed, first CWT analysis was performed for maximum resolution and to create a reference. Analysis is performed in MATLAB using the Wavelet Toolbox. For the analyzing wavelet Analytic Morse Wavelet was chosen among the available wavelets in the toolbox [32]. This is because it has more tunable parameters according the needs of the application and by tuning similar behavior to the other continuous wavelets can be obtained [33]. Approximate center frequency of the mother wavelet is tuned according to fundamental harmonic as well as expected harmonic and inharmonic partials of the played pitches. Time bandwidth parameter $\left(p^{2}\right)$ that corresponds to width of the wavelet in the time domain and symmetry parameter $(\gamma)$ that corresponds to distribution around the peak frequency of wavelet tuned to 120 and to 3 respectively for best resolution within the computational limits of the used computer. 20 voices per octave was set for frequency resolution. To be able to present the 3D time-scale plots of the data, energy of every scale is calculated and the first 100 scales that have the most energy are used for graphics. For more intuitive evaluation, scale values are converted to approximate center frequencies. For convenience, only the graphics of pitch A3 from bridge and hole sensors, played in both finger position with each guitar is presented in Figure 4 in the main text. Graphics of other pitches can be found in Appendix. For the rest of the text, the mass produced guitar will be referred as "Guitar 1" and the luthier-made guitar will be referred as "Guitar 2".

CWT is a computationally expensive method for general applicability and its resolution can be far more detailed for some applications in the area. For this reason, WPT is performed to present a more affordable solution that provides adequate detail. WPT is computed for 10 levels which corresponds to a minimum frequency bandwidth of $32 \mathrm{~Hz}$. Daubechies Wavelet with 4 vanishing moments is chosen because while the lower vanishing moments providing better time resolution, high vanishing moments (smoother wavelets) provide better frequency resolution. Since WPT is not redundant and have lower resolution, data at the output was smaller than the CWT. This allowed the using first 125 frequencies with the most energy in the graphics. In addition, because the resulted waveforms are discrete there were no need to present the graphics in 3D. Analysis of pitch A3 is presented in Figure 5 and the rest is presented in the Appendix.

\section{RESULTS}

Before making remarks on the guitars, it should be stated that results clearly support the superiority of wavelet analysis over Fourier and Short Time Fourier Transform. It can be seen that localization at the low frequencies is in a degree that can't be reached using STFT. Partials as low as $30 \mathrm{~Hz}$ are observable. Also, wavelet analysis can present non-harmonic partials more accurately. For example, in the Figure 4, partial $660 \mathrm{~Hz}$ can be seen even though it is not a harmonic of pitch A3 since, 660 $\mathrm{Hz}$ is not an even multiply of $220 \mathrm{~Hz}$. By comparing Figure 4(a) and (b), it can be observed that most prominent higher partials of bridge portion are approximately at $660 \mathrm{~Hz}$ and 880 $\mathrm{Hz}$ and even those don't have a high energy ratio relative to the formant at $220 \mathrm{~Hz}$ whereas in the hole area, there are not only larger number of prominent partials which are at $440 \mathrm{~Hz}$, $660 \mathrm{~Hz}, 880 \mathrm{~Hz}$ and $1760 \mathrm{~Hz}$; energy intensity of these partials are higher than their bridge counterparts. One interesting evidence is that the formant at $220 \mathrm{~Hz}$ has lower energy while the harmonic at $880 \mathrm{~Hz}$ having the most energy among the partials. Usually, it is expected for low frequencies to have more energy in around air holes because they prevent phase cancellation. In this case considering the effect of air coupling is more present around the air cavity, this shows low frequencies created by the back plate and top plate are cancelled each other. If one investigates the same aspects in Guitar 2 using the Figure 4 (e) and (f), they can see that partials as well as energy distribution among them is similar between hole and bridge areas. A comparison that can be make using this evaluation is the body vibrations are more homogenous in Guitar 2 than they are in Guitar 1, which points to the considerations in choosing the materials in top plate and back plate. If one observes the effect of fingering position comparing Figure 4 (a) to (c) and Figure 4 (b) to (d) for Guitar 1: while the frequency location of partials almost similar there are slight differences between time envelopes. Same evaluations can be made for Guitar 2 by comparing Figure 4 (e) to (g) and (f) to (h). This implicates that effect of fingering on the overall timbre of guitar is negligible. It is observed that distribution of energy in all the graphics of Guitar 1, it can be seen that energy is spread around the center frequencies of partials. In Guitar 2, energy is concentrated around the centers. When one looks at the time envelopes in Guitar 1, within the individual partials, energy is mostly concentrated towards to attack portion which makes the overall sound perceived more intense while making damping portion shorter. Another observation is that the instantaneous frequency of the formant matches to its tuning frequency almost instantly. If same aspects are investigated in Guitar 2, it can be seen that energy of partials have a more even distribution among the time portions with the relatively low intensity in the attack and longer damping time. Also, steady state portions of the partials are more prominent. However, there is a distinguishable time for instantaneous frequency to match its tuning frequency which can be explained by Guitar 1 being much older than Guitar 2 and the woods used in musical instruments need time to mature for reaching its ideal sound characteristics. Another observation that can be made using figures in Appendix is that the frequency range of Guitar 1 is narrower with the highest 
partial being at around $3900 \mathrm{~Hz}$ while it is around $5900 \mathrm{~Hz}$ in Guitar 2. In general, it can be said that, Guitar 1 has simpler timbre with low sustain and low definition whereas Guitar 2 has richer and cleaner timbre with higher sustain and higher

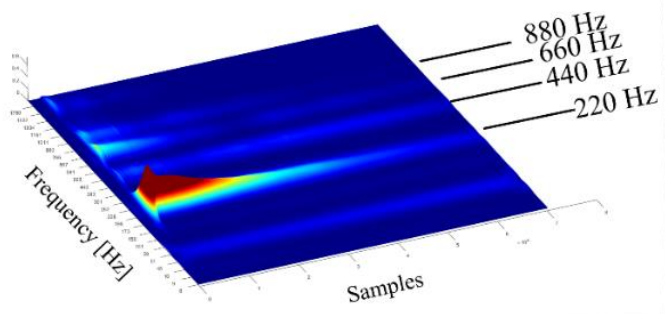

(a) Guitar 1, open position, bridge sensor

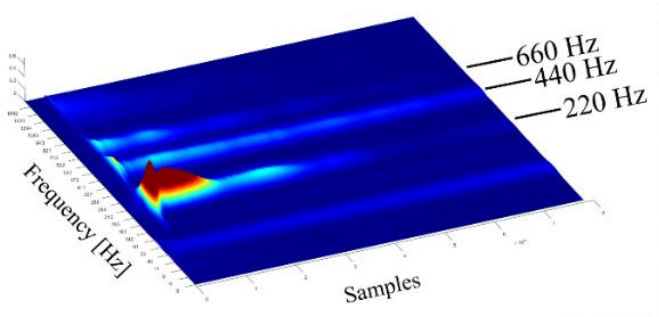

(c) Guitar 1, 5th position, bridge sensor

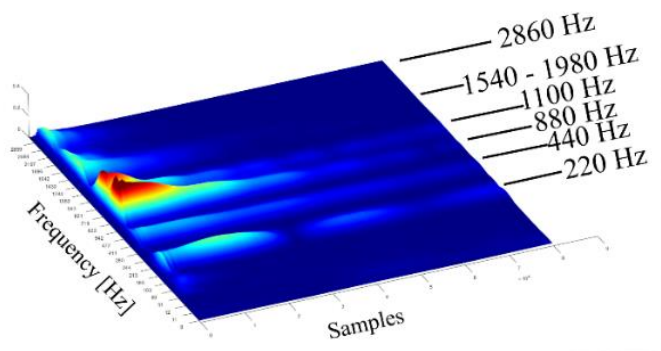

(e) Guitar 2, open position, bridge sensor

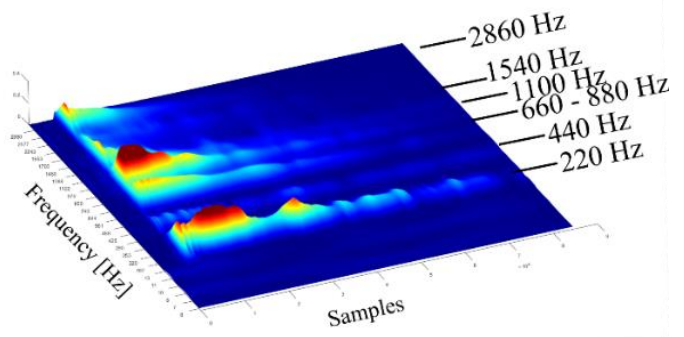

(g) Guitar 2, 5th position, bridge sensor definition. Also, Guitar 2 has more number of higher frequency partials than Guitar 1, making its timbre what is called "bright".

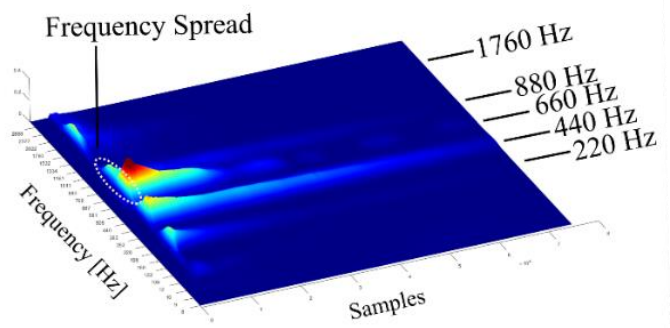

(b) Guitar 1, open position, hole sensor

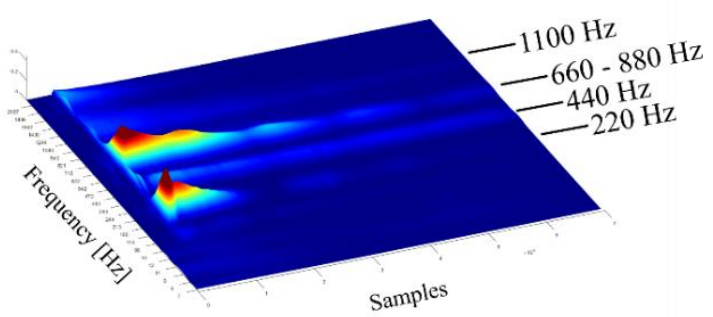

(d) Guitar 1, 5th position, hole sensor

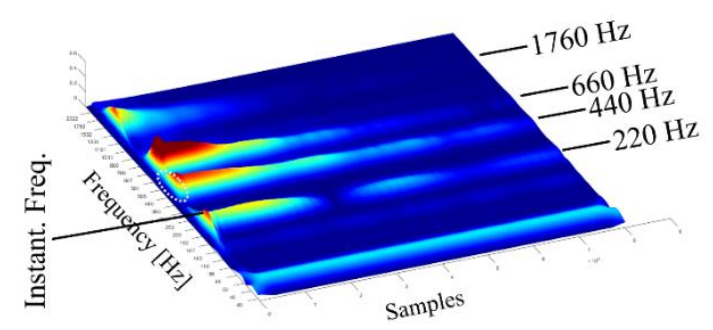

(f) Guitar 2, open position, hole sensor

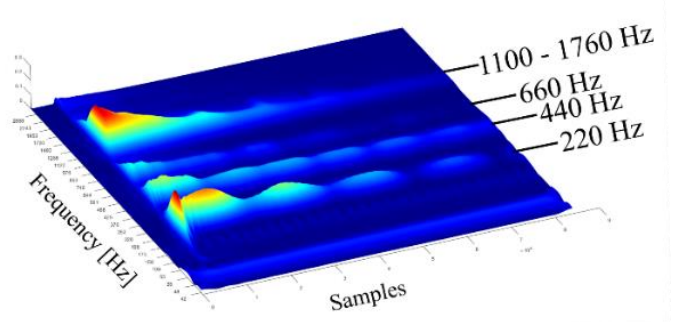

(h) Guitar 2, 5th position, hole sensor

Figure 4. CWT results of A3

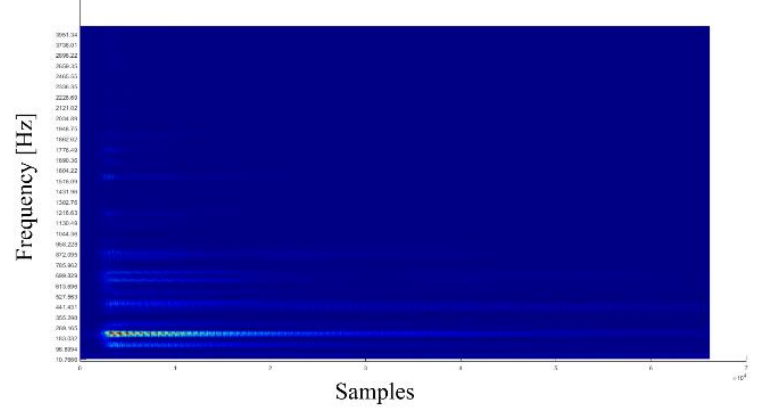

(a) Guitar 1, open position, bridge sensor

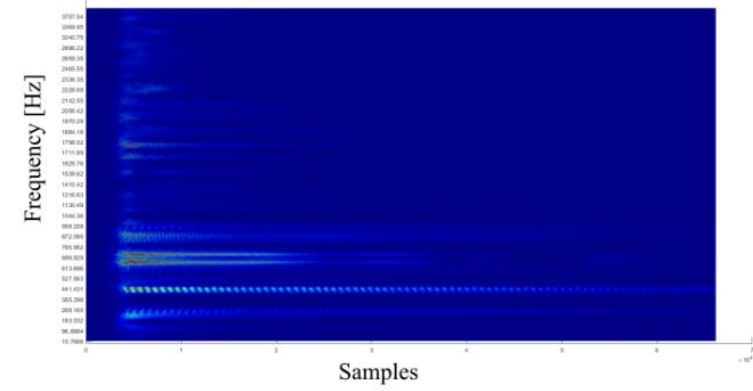

(b) Guitar 1, open position, hole sensor 


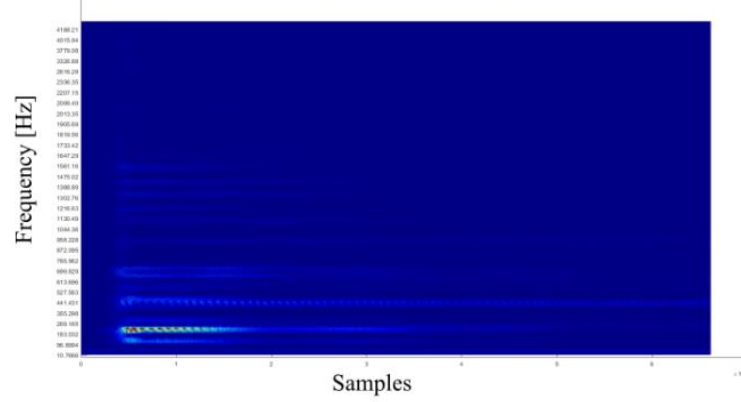

(c) Guitar 1, 5th position, bridge sensor

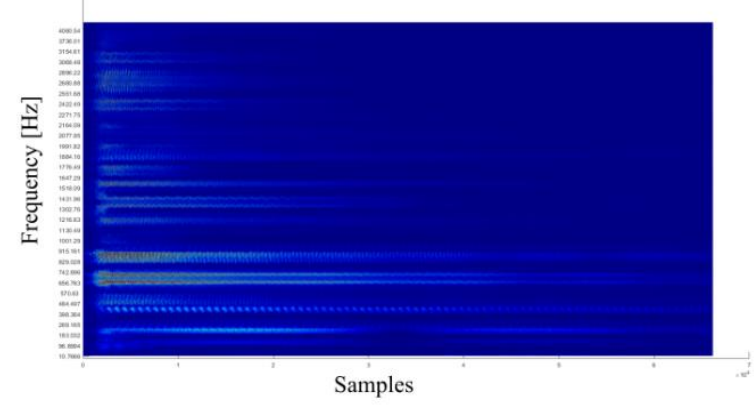

(e) Guitar 2, open position, bridge sensor

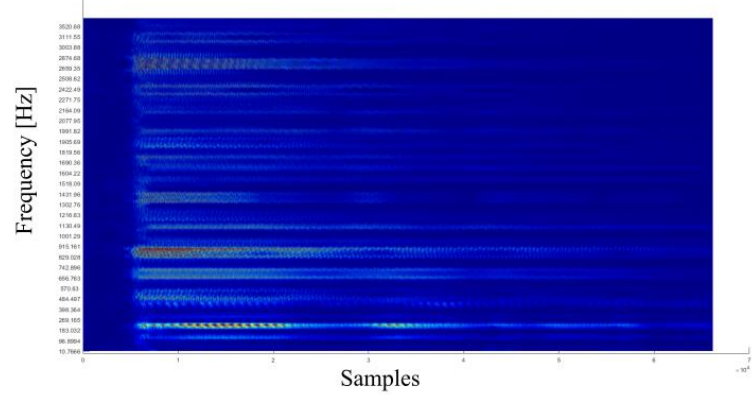

(g) Guitar 2, 5th position, bridge sensor

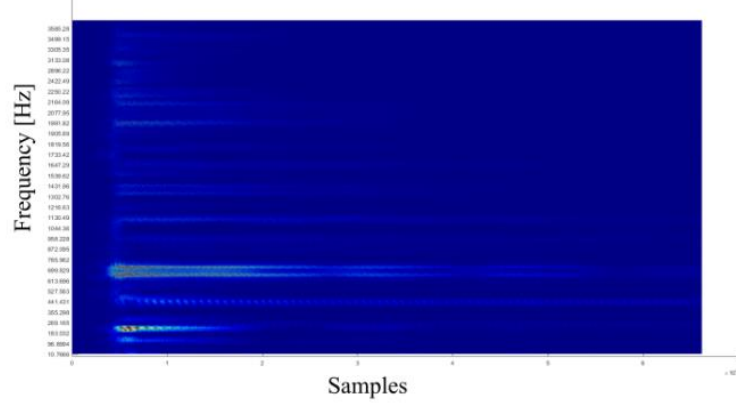

(d) Guitar 1, 5th position, hole sensor

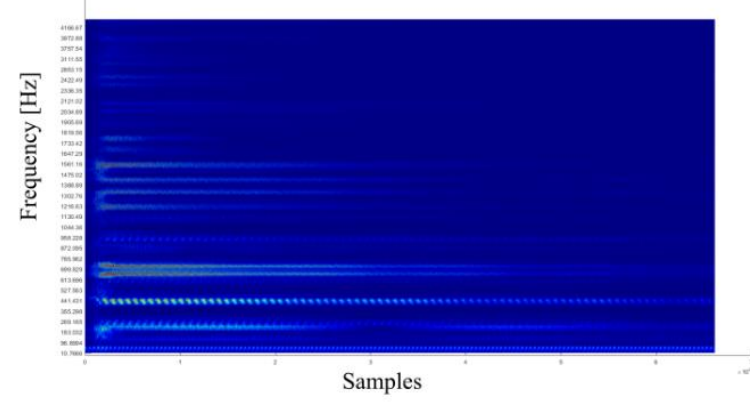

(f) Guitar 2, open position, hole sensor

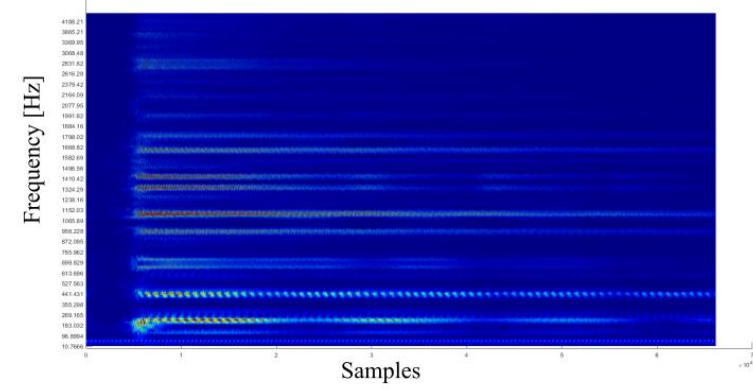

(h) Guitar 2, 5th position, hole sensor

Figure 5. WPT results of A3

\section{CONCLUSION AND DISCUSSION}

A complete system for investigating timbral qualities of guitars is proposed in this paper. A practical data capturing setup by employing piezo-film sensors with conventional playing position and excitement using pluck is presented. Data analysis is performed using Continuous Wavelet Transform for a high-resolution analysis and a computationally effective alternative is presented with Wavelet Packet Transform. Results show detailed presentation of harmonic and inharmonic partials as well as time envelopes of them in a resolution that cannot be obtainable using Short-Time Fourier Transform. Results allow for objective analysis on the timbre of a guitar as well as for comparison between the timbral properties of the two guitars. A disadvantage of the system is not being able to obtain modal shapes of guitar bodies. Modal shapes are generally requested for the detailed analysis on the resonant frequencies of materials and the proposed system can already provide this information so, the modal shapes are no longer necessary for that purpose. If one still needs to obtain modal shapes, they have to resort aforementioned Chladni, holographic or Digital Image Correlation techniques.

Author is well aware of that with its current state proposed system is not ready for general usage. Boundaries of this study is mainly determined by the budget and time limitations. Nevertheless, it is firmly believed that suggested work frame initiates a powerful system for analyzing timbral qualities of guitar while undoubtedly contributing to the literature on the applications of wavelet transform on micro level analysis of musical signals. For further study more sensors with some of them on the back plate of guitar can be used to provide broader analysis. Test can be performed at each iterations of guitar making process which in turn makes it possible to observe the effects of design parameters. Study can be performed on large number of guitars which will provide a way for categorization of guitar designs.

\section{REFERENCES}

[1] Rossing, T.D., Moore, F.R., Wheeler, P.A. (2002). The science of sound. Pearson, pp. 208-210

[2] Chladni, E.F.F. (2015). Treatise on Acoustics: The First Comprehensive English Translation of EFF Chladni's Traité d'Acoustique. Springer.

[3] Rossing, T.D., Eban, G. (1999). Normal modes of a radially braced guitar determined by electronic TV holography. The Journal of the Acoustical Society of 
America,

$106(5)$ :

2991-2996.

https://doi.org/10.1121/1.428118

[4] Curtu, I., Stanciu, M.D., Cretu, N., Rosca, I. (2009). Modal analysis of different types of classical guitar bodies. Proceedings of the 10th WSEAS International Conference on Acoustics \& Music: Theory \& Applications-AMTA09 (ISTP/ISI Proceeding of Thomson Scientific-Institute for Scientific Information, WisconsinUnited States, pp. 23-25.

[5] Patil, K., Baqersad, J., Ludwigsen, D., Dong, Y. (2016). Extracting vibration characteristics of a guitar using finite element, modal analysis, and digital image correlation techniques. In Proceedings of Meetings on Acoustics 172ASA, 29(1): 065003. https://doi.org/10.1121/2.0000465

[6] Woodhouse, J. (2004). Plucked guitar transients: Comparison of measurements and synthesis. Acta Acustica united with Acustica, 90(5): 945-965.

[7] American National Standards Institute, American National Psychoacoustical Terminology. S3.20, Acoustical Society of America (ASA), New York, NY, USA, 1973. pp. 29, 30, 41.

[8] Richardson, E.G. (1954). The transient tones of wind instruments. The Journal of the Acoustical Society of America, 26(6): 960-962.

https://doi.org/10.1121/1.1907460

[9] Berger, K.W. (1964). Some factors in the recognition of timbre. The Journal of the Acoustical Society of America, 36(10): 1888-1891. https://doi.org/10.1121/1.1919287

[10] Craig, J.H., Jeffress, L.A. (1962). Effect of phase on the quality of a two-component tone. The Journal of the Acoustical Society of America, 34(11): 1752-1760. https://doi.org/10.1121/1.1909118

[11] Grey, J.M. (1977). Multidimensional perceptual scaling of musical timbres. The Journal of the Acoustical Society of $\quad$ America, $1270-1277$ https://doi.org/10.1121/1.381428

[12] Ballora, M. (2003). Essentials of Music Technology. Upper Saddle River: Prentice Hall.

[13] Weeks, M. (2010). Digital Signal Processing Using MATLAB \& Wavelets. Jones \& Bartlett Publishers.

[14] Gargour, C., Gabrea, M., Ramachandran, V., Lina, J.M. (2009). A short introduction to wavelets and their applications. IEEE Circuits and Systems Magazine, 9(2): 57-68. https://doi.org/10.1109/MCAS.2009.932556

[15] Gabor, D. (1946). Theory of communication. Part 1: The analysis of information. Journal of the Institution of Electrical Engineers-Part III: Radio and Communication Engineering, 93(26): 429-441.

[16] Meyer, Y. (1989). Orthonormal wavelets. Wavelets, 2137. https://doi.org/10.1007/978-3-642-97177-8_2

[17] Mallat, S.G. (1989). A theory for multiresolution signal decomposition: The wavelet representation. IEEE Transactions on Pattern Analysis and Machine Intelligence, 11(7): 674-693. https://doi.org/10.1109/34.192463

[18] Daubechies, I. (1988). Orthonormal bases of compactly supported wavelets. Communications on Pure and Applied Mathematics, 41(7): 909-996. https://doi.org/10.1002/cpa.3160410705

[19] Daubechies, I. (1990). The wavelet transform, timefrequency localization and signal analysis. IEEE Transactions on Information Theory, 36(5): 961-1005. https://doi.org/10.1109/18.57199
[20] Daubechies, I. (1993). Orthonormal bases of compactly supported wavelets II. Variations on a theme. SIAM Journal on Mathematical Analysis, 24(2): 499-519. https://doi.org/10.1137/0524031

[21] Beylkin, G., Coifman, R., Rokhlin, V. (1991). Fast wavelet transforms and numerical algorithms I. Communications on Pure and Applied Mathematics, 44(2): 141-183. https://doi.org/10.1002/cpa.3160440202

[22] Meyer, Y. (1998). Wavelets, Vibrations and Scalings (No. 9). American Mathematical Soc.

[23] Wickerhauser, M.V. (1996). Adapted Wavelet Analysis: From Theory to Software. CRC Press.

[24] Alm, J.F., Walker, J.S. (2002). Time-frequency analysis of musical instruments. SIAM Review, 44(3): 457-476. https://doi.org/10.1137/S00361445003822

[25] Pielemeier, W.J., Wakefield, G.H., Simoni, M.H. (1996). Time-frequency analysis of musical signals. Proceedings of the IEEE, 84(9): 1216-1230. https://doi.org/10.1109/5.535242

[26] Salimpour, Y., Abolhassani, M.D. (2006). Auditory wavelet transform based on auditory wavelet families. 2006 International Conference of the IEEE Engineering in Medicine and Biology Society, New York, NY, USA, pp. $1731-1734$ https://doi.org/10.1109/IEMBS.2006.260717

[27] Dutilleux, P., Grossmann, A., Kronland-Martinet, R. (1988). Application of the wavelet transform to the analysis, transformation and synthesis of musical sounds. Audio Engineering Society Convention 85. Audio Engineering Society.

[28] Kostek, B., Zwan, P., Dziubinski, M. (2002). Statistical analysis of musical sound features derived from wavelet representation. Audio Engineering Society Convention 112. Audio Engineering Society.

[29] Beltrán, J.R., Beltrán, F. (2003). Additive synthesis based on the continuous wavelet transform: A sinusoidal plus transient model. DAFX03, 3: 1-6.

[30] Connectivity, T.E. (2017). SDT Shielded Piezo Sensors. SDT1 datasheet, Julio.

[31] National Instruments Corp. Measuring Vibrations with Accelerometers. https://www.ni.com/entr/innovations/white-papers/06/measuring-vibrationwith-accelerometers, accessed on Jun. 5, 2020.

[32] Misiti, M., Misiti, Y., Oppenheim, G., Poggi, J.M. (1996). Matlab wavelet toolbox user's guide. The MathWorks Inc., Natick, MA, USA, version, 2.

[33] Lilly, J.M., Olhede, S.C. (2012). Generalized Morse wavelets as a superfamily of analytic wavelets. IEEE Transactions on Signal Processing, 60(11): 6036-6041. https://doi.org/10.1109/TSP.2012.2210890

\section{NOMENCLATURE}

$\begin{array}{ll}t & \text { Time } \\ f & \text { Frequency } \\ \Delta t & \text { Time resolution } \\ \Delta f & \text { Frequency resolution } \\ \psi_{a, b} & \text { Wavelet } \\ V & \text { Vector Space } \\ V_{1}, V_{2} & \text { Vector Subspaces } \\ p^{2} & \text { Time bandwidth } \\ \gamma & \text { Symmetry parameter }\end{array}$




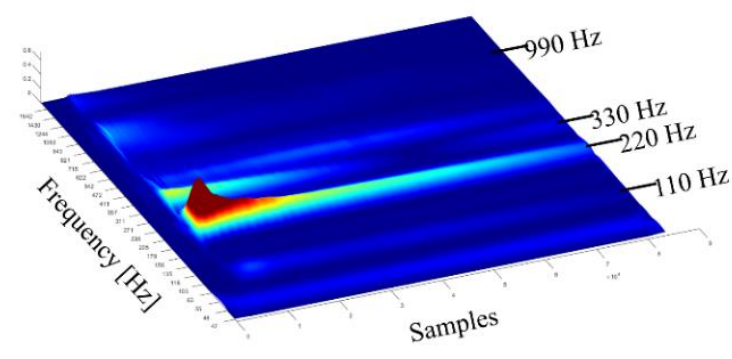

(a) Guitar 1, open position, bridge sensor

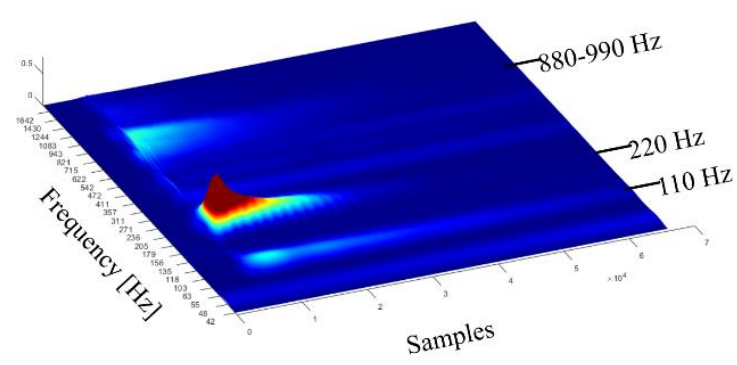

(c) Guitar 1, 5th position, bridge sensor

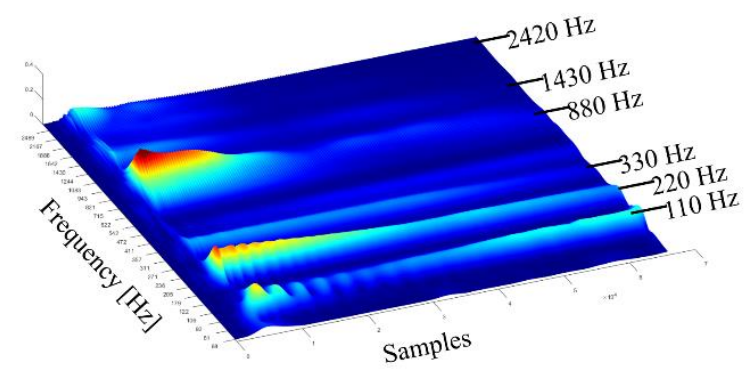

(e) Guitar 2, open position, bridge sensor

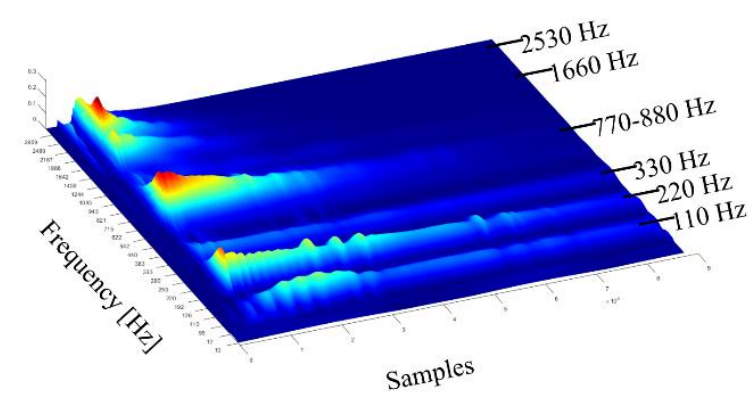

(g) Guitar 2, 5th position, bridge sensor

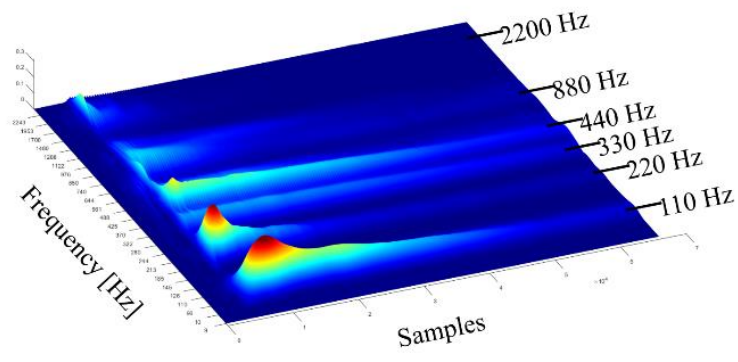

(b) Guitar 1, open position, hole sensor

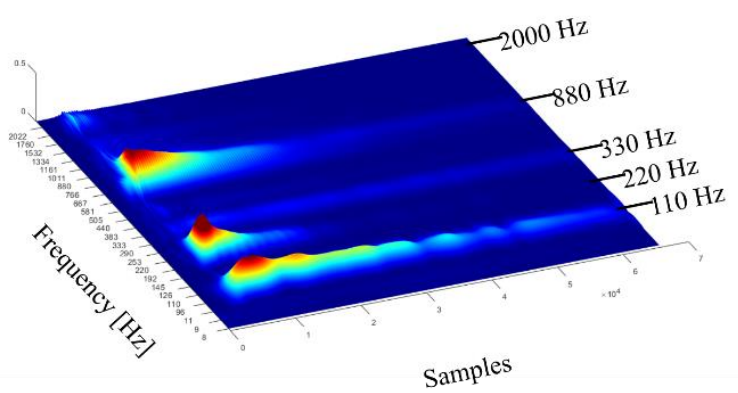

(d) Guitar 1, 5th position, hole sensor

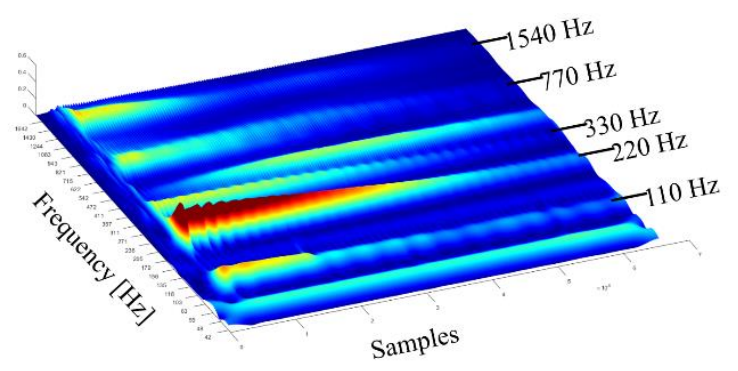

(f) Guitar 2, open position, hole sensor

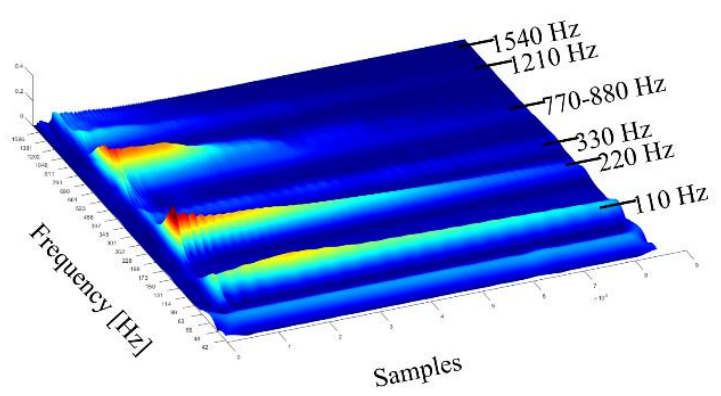

(h) Guitar 2, 5th position, hole sensor

Figure A1. CWT results of pitch A2

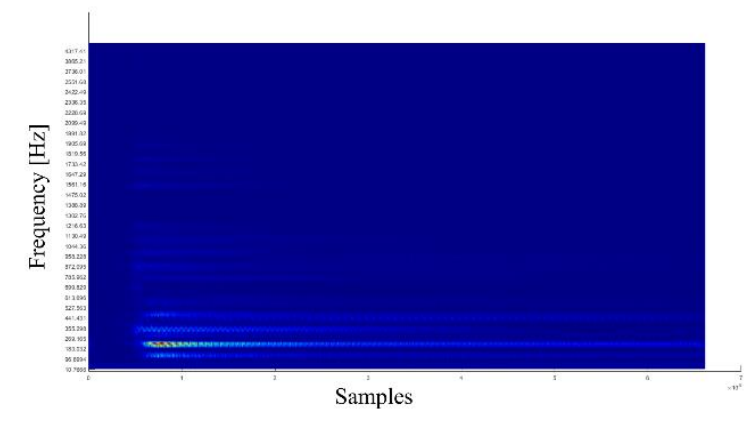

(a) Guitar 1, open position, bridge sensor

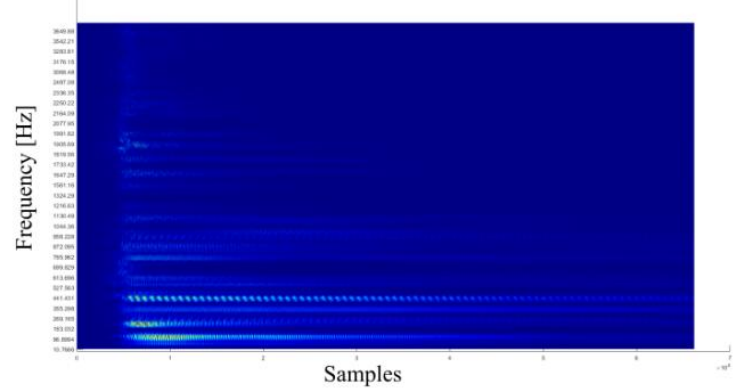

(b) Guitar 1, open position, hole sensor 


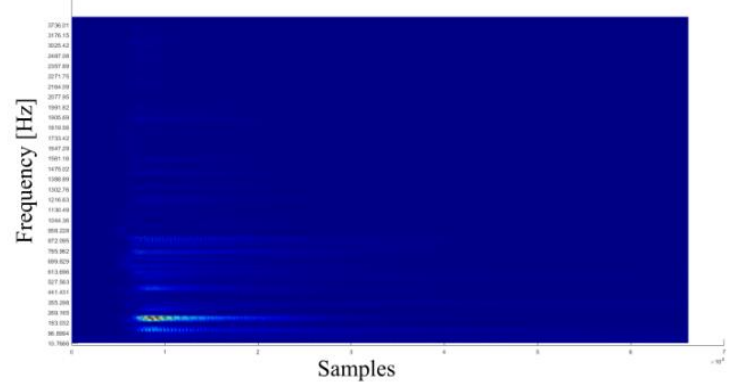

(c) Guitar 1, 5th position, bridge sensor

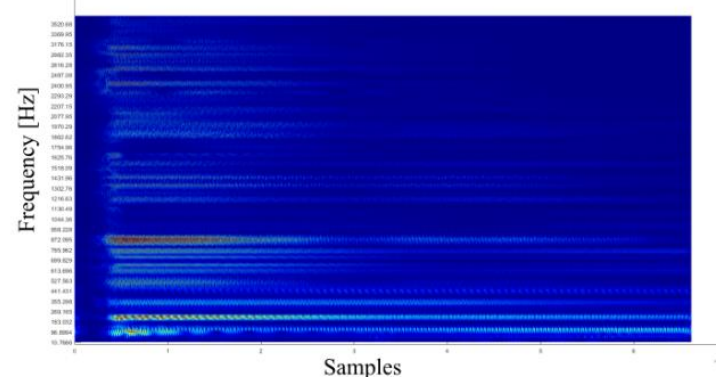

(e) Guitar 2, open position, bridge sensor

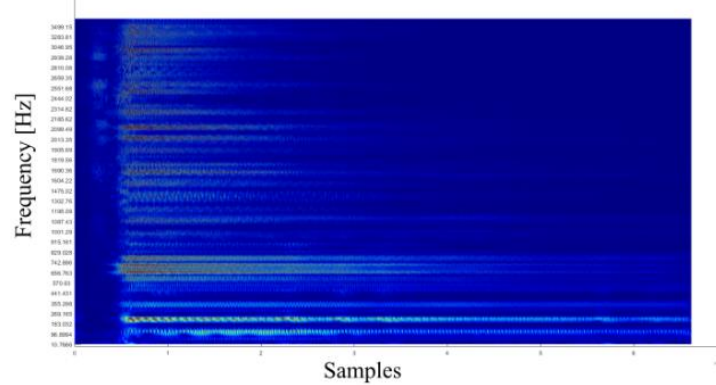

(g) Guitar 2, 5th position, bridge sensor

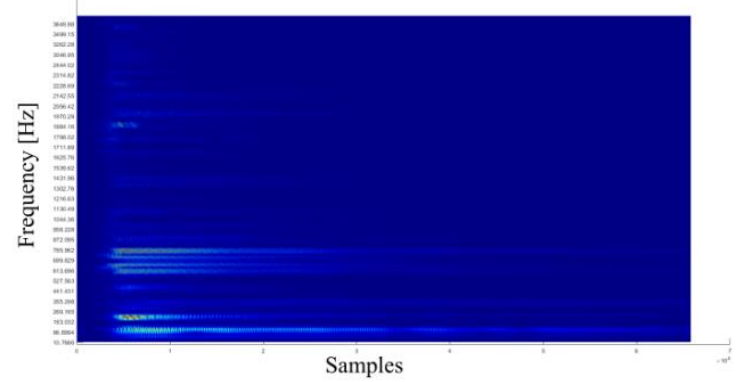

(d) Guitar 1, 5th position, hole sensor

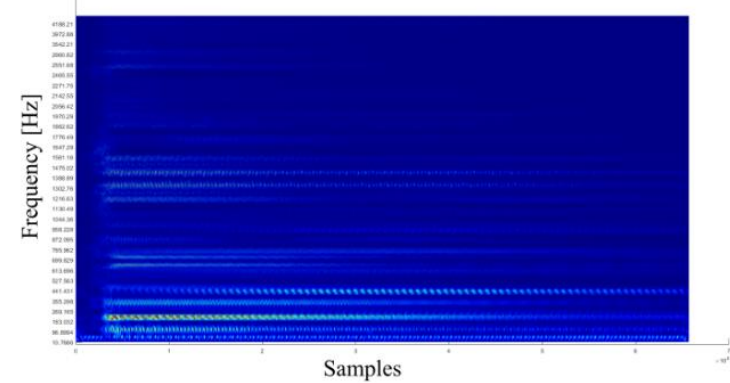

(f) Guitar 2, open position, hole sensor

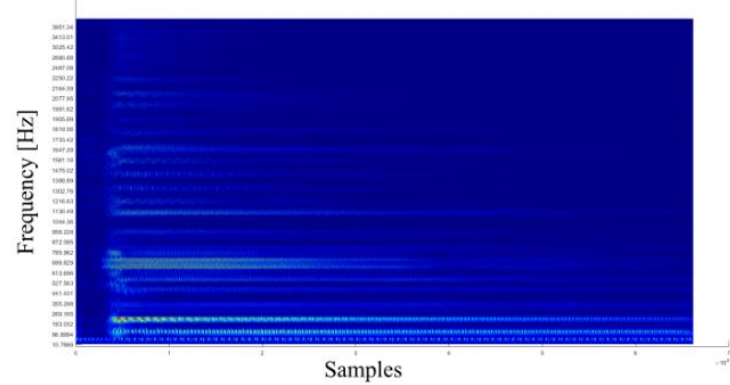

(h) Guitar 2, 5th position, hole sensor

Figure A2. WPT results of pitch A2

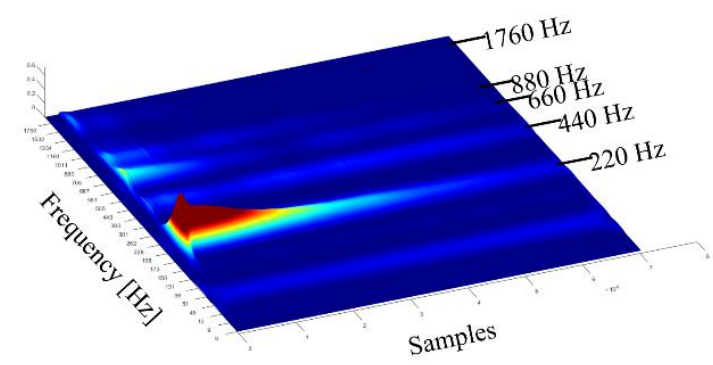

(a) Guitar 1, open position, bridge sensor

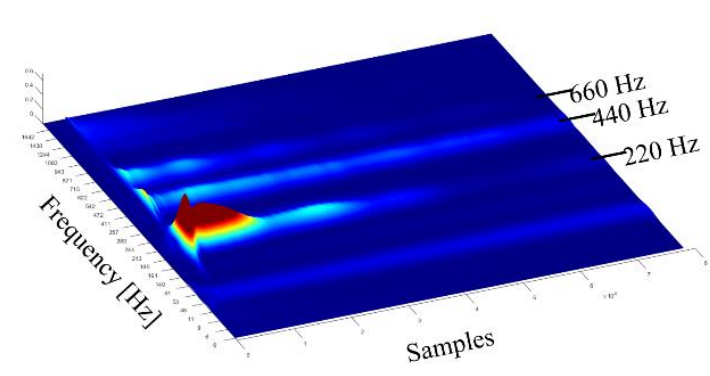

(c) Guitar 1, 5th position, bridge sensor

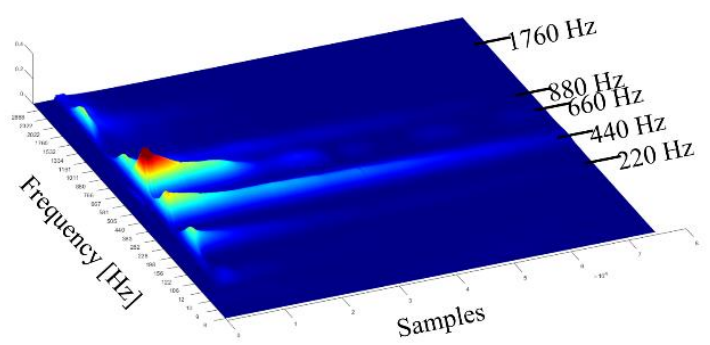

(b) Guitar 1, open position, hole sensor

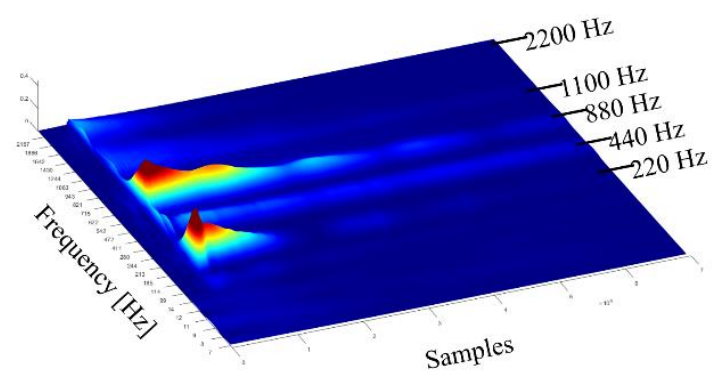

(d) Guitar 1, 5th position, hole sensor 


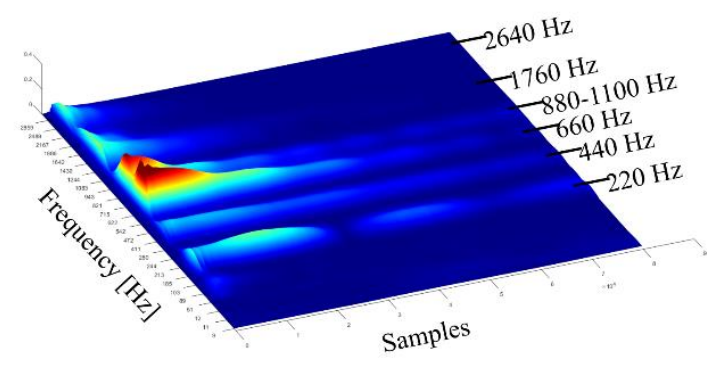

(e) Guitar 2, open position, bridge sensor

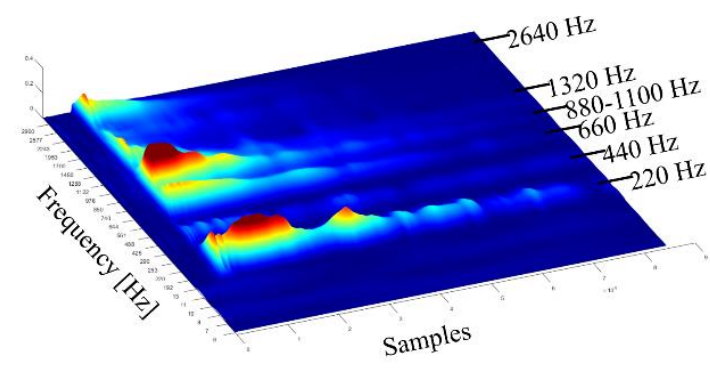

(g) Guitar 2, 5th position, bridge sensor

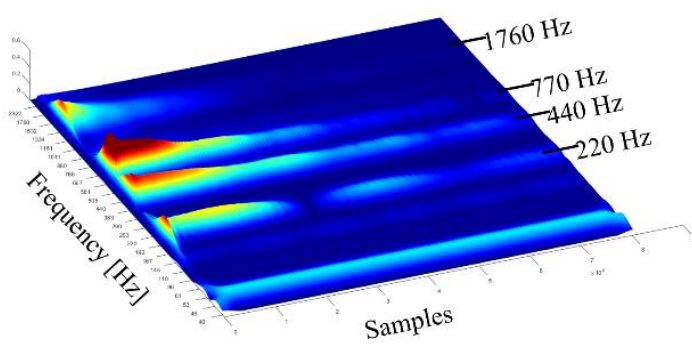

(f) Guitar 2, open position, hole sensor

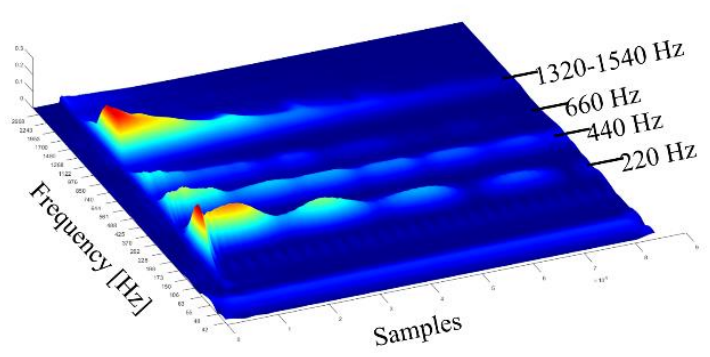

(h) Guitar 2, 5th position, hole sensor

Figure A3. CWT results of pitch A4

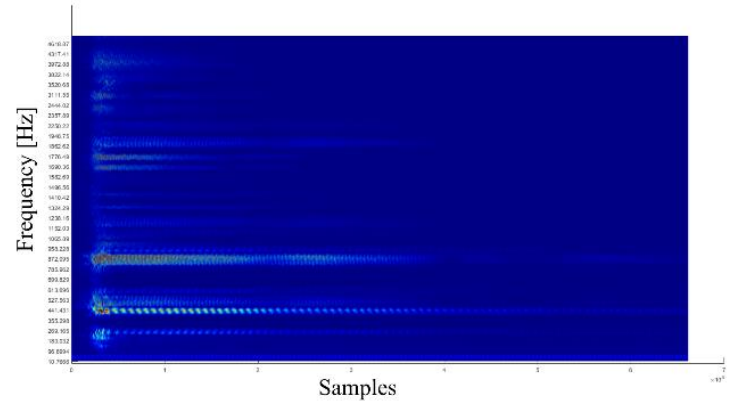

(a) Guitar 1, open position, bridge sensor

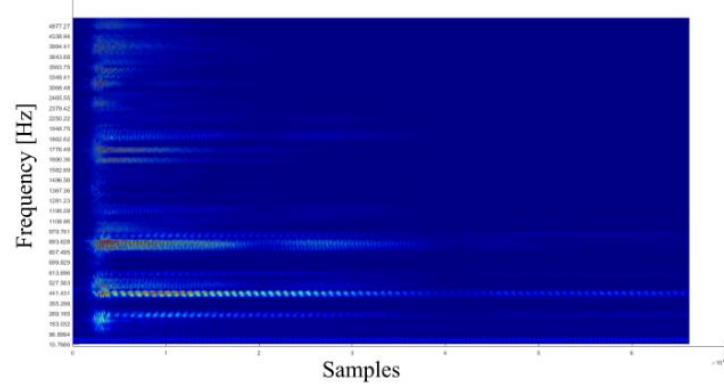

(c) Guitar 1, 5th position, bridge sensor

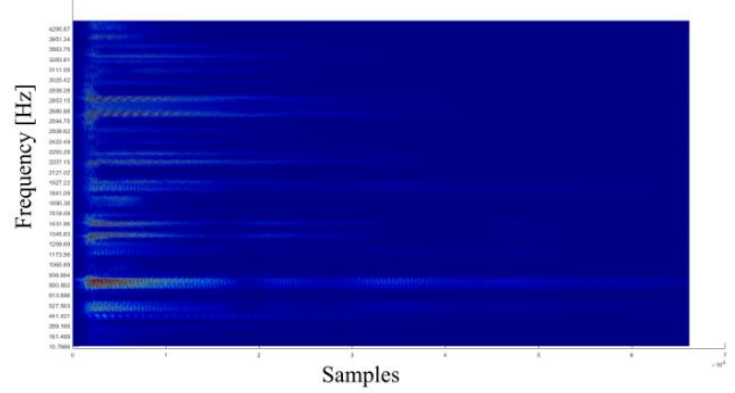

(e) Guitar 2, open position, bridge sensor

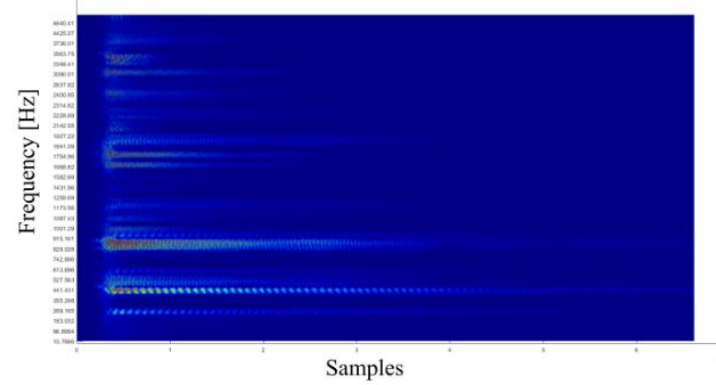

(b) Guitar 1, open position, hole sensor

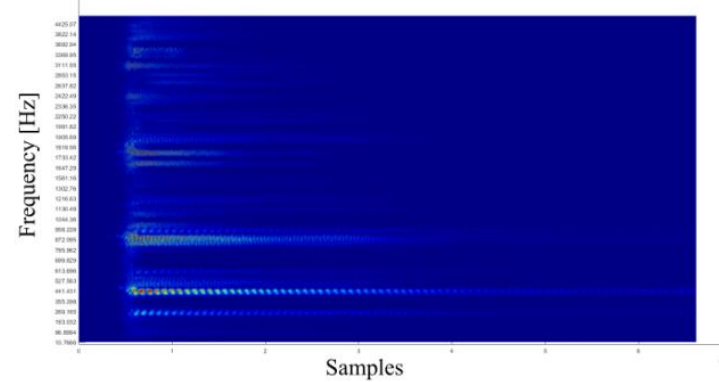

(d) Guitar 1, 5th position, hole sensor

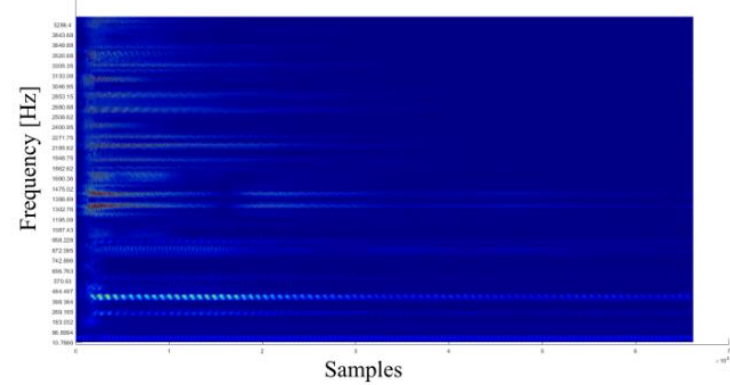

(f) Guitar 2, open position, hole sensor 


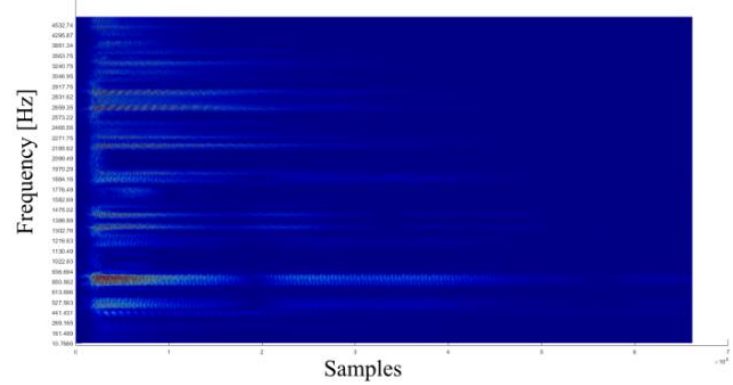

(g) Guitar 2, 5th position, bridge sensor

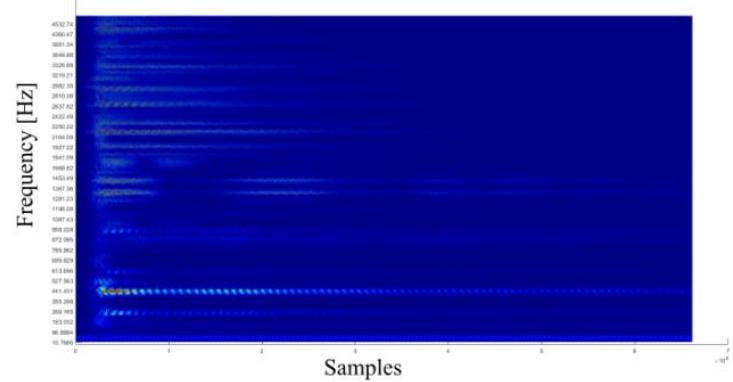

(h) Guitar 2, 5th position, hole sensor

Figure A4. WPT results of pitch A4 